\title{
Stable monotone iterative solutions to a class of bound- ary value problems of nonlinear fractional order differential equations
}

\author{
Sajjad Alia ${ }^{\mathrm{a}}$ Muhammad Arifa ${ }^{\mathrm{a}}$, Durdana Lateef ${ }^{\mathrm{b}, *}$, Mohammad Akram $^{\mathrm{c}}$ \\ ${ }^{a}$ Department of Mathematics, Abdul Wali Khan University of Mardan, Khyber Pakhtunkhwa, Pakistan. \\ ${ }^{b}$ Department of Mathematics, College of Science, Taibah University, Madinah, KSA. \\ ${ }^{c}$ Department of Mathematics, Faculty of Science, Islamic University of Madinah, Madinah, KSA.
}

\begin{abstract}
We construct sufficient conditions for existence of extremal solutions to boundary value problem (BVP) of nonlinear fractional order differential equations (NFDEs). By combing the method of lower and upper solution with the monotone iterative technique, we construct sufficient conditions for the iterative solutions to the problem under consideration. Some proper results related to Hyers-Ulam type stability are investigated. Base on the proposed method, we construct minimal and maximal solutions for the proposed problem. We also construct and provide maximum error estimates and test the obtain results by two examples.
\end{abstract}

Keywords: Nonlinear fractional differential equations, iterative technique, upper and lower solutions, uniqueness and existence.

2010 MSC: 34A08, 34A45.

(C)2019 All rights reserved.

\section{Introduction}

The work of this manuscript is to devoted to the investigation of sufficient conditions for existence of extremal solutions to the following BVP of NFDEs

$$
\mathrm{D}^{\mathrm{p}} v(\mathrm{t})+\mathrm{k}(\mathrm{t}, v(\mathrm{t}))=0,0<\mathrm{t}<1,3<\mathrm{p} \leqslant 4, \quad v(0)=v^{\prime}(0)=v^{\prime \prime}(0)=v^{\prime \prime}(1)=0,
$$

where $v \in \mathrm{C}[0,1]$ and $\mathrm{k}: \mathrm{I} \times \mathbb{R} \rightarrow \mathbb{R}$.

In recent decades, it has become a significant fact that FDEs are powerful tools in modeling the various phenomena in sciences and engineering such as biology, economics, mechanics and physics. For many applications of NFDEs, see $[1,2,8,14,17,24,25,29]$ and the references cited therein. Due to this fact, the

\footnotetext{
${ }^{*}$ Corresponding author

Email addresses: sajjad_ali@sbbu.edu.pk (Sajjad Ali), marifmaths@awkum.edu.pk (Muhammad Arif), drdurdanamath@gmail.com (Durdana Lateef), akramkhan_20@rediffmail.com (Mohammad Akram)

doi: $10.22436 /$ jnsa.012.06.04
}

Received: 2018-09-16 Revised: 2018-11-16 Accepted: 2018-12-14 
area devoted to NFDEs has been given great attention by most of the researchers. In recent decade, there has been significant work of research on empirical questions of the existence/uniqueness of solutions (or positive solutions) to large numbers of BVPs of FDEs, (see for detail [4, 12, 15, 30, 32, 33, 36, 38, 39]). In the aforesaid work researchers used classical fixed point theory to develop the existence theory of solutions to BVPs of NFDEs.

On the other hand one important aspect of existence theory is devoted to the iterative solutions of FDEs with initial and boundary conditions. The aforesaid area has been very well explored for classical differential equations. However for FDEs, this area need further exploration. In last few years some works devoted to the extremal solutions of NFDEs with boundary and initial conditions were carried out, which we refer in $[6,7,18,23,37,40]$. In the cited papers, the authors used coupled of monotone iterative technique with the upper and lower solutions method to obtain results for approximating the solutions of NFDEs. The aforesaid scheme has been considered by many authors for NFDEs with boundary conditions. For instance, Shah and his co-author [31] applied the aforementioned method to study a coupled system of NFDEs with three point boundary conditions. A similar attempt was made by Khan [16]. He developed the aforesaid scheme for the following class of NFDE

$$
\left\{\begin{array}{l}
\mathrm{D}^{\alpha} v(\mathrm{t})+\mathrm{f}(\mathrm{t}, x(\mathrm{t}))=0, \mathrm{t} \in(0,1), 1<\alpha<2, \\
\left.v^{\prime}(\mathrm{t})\right|_{\mathrm{t}=0}=0, v(1)=\xi x(\eta),
\end{array}\right.
$$

where $\xi, \eta \in(0,1)$.

Recently another aspect has greatly attracted the attentions of researchers known as stability analysis. The mentioned aspect has been very well studied for classical differential equations. Various kinds of stability including exponential stability, Laypunove stability, etc have been introduced so far for both classical and FDEs. In last few years Ulam type stability has been considered in many articles for classical as well as FDEs. But the respective stability has been very well investigated mostly for initial value problems of FDEs and rarely studied for BVPs of FDEs, for detail see [21, 26-28, 34]. Motivated by the aforementioned works, our purpose in this paper is to study the existence of upper and lower solutions and to find conditions for iterative solutions to NFDEs (1.1). We compute the error estimates to the problem under consideration by using the monotone iterative method combine with the method of upper and lower solution. We also investigate different kinds of Ulam stability for the iterative solutions of the considered problem. We provide two example to illustrate our work. The physical behavior of the upper and lower solutions has been provided in various plots by using Matlab.

\section{Preliminaries}

In this section, we present some basic definitions and known lemmas that are supportive to the proof of our main results, which can be found in $[14,17,24,25,33,37,39]$.

Definition 2.1. The Riemann-Liouville fractional integral of order $\delta \in \mathbb{R}_{+}$of a function $\phi \in L([0,1], \mathbb{R})$ is defined by

$$
I^{\delta} \phi(t)=\frac{1}{\Gamma(\delta)} \int_{0}^{t}(t-\rho)^{\delta-1} \phi(\rho) d \rho,
$$

provided that on right side's integral is pointwise defined.

Definition 2.2. Let $v(t) \in \mathrm{C}^{\mathrm{n}}[\mathrm{a}, \mathrm{b}]$ be the function in $\mathrm{t}$, then the Riemann-Liouville derivative of the fractional order " $p$ ", where $p \in \mathcal{R}^{+}$is defined as

$$
D_{a}^{p} v(t)=\frac{1}{\Gamma(n-p)}\left(\frac{d}{d t}\right)^{n} \int_{a}^{t}(t-\lambda)^{n-p-1} v(\lambda) d \lambda, n-1 \leqslant p \leqslant n, n \in N,
$$

provided that integral on the right hand side is defined point wise on $(a, \infty)$, when $n=[p]+1$. In situation, when $p \in Z^{+}$and $n=p$, then derivative of fractional order coincides with traditional derivative of integer order. 
Definition 2.3. Choose $W=C[0,1]$ be a Banach space under the norm $\|v\|=\max _{t \in[0,1]}|v(t)|$ and assume that $\mathrm{V} \subset \mathrm{W}$ which is satisfies the partially ordering property. The operator $\mathrm{T}: \mathrm{V} \rightarrow \mathrm{U}$ is known as nondecreasing if for each $v_{1}, v_{2} \in \mathrm{V}$ and $v_{1} \leqslant v_{2}$ gives $T v_{1} \leqslant T v_{2}$. The operator $T$ is known as non increasing if for each $v_{1}, v_{2} \in \mathrm{V}$ and $v_{1} \leqslant v_{2}$ gives $\mathrm{T} v_{1} \geqslant \mathrm{~T} v_{2}$.

Definition 2.4. Let $(\mathrm{I}-\mathrm{T}) v_{1} \leqslant 0$ for an operator equation $(\mathrm{I}-\mathrm{T}) v=0$, then $v_{1} \in \mathrm{U}$ is known as a lower solution of $(\mathrm{I}-\mathrm{T}) v=0$ and $(\mathrm{I}-\mathrm{T}) v_{2} \geqslant 0$ for an operator equation $(\mathrm{I}-\mathrm{T}) \boldsymbol{v}=0$, then $v_{2} \in \mathrm{U}$ is known as an upper solution of $(\mathrm{I}-\mathrm{T}) v=0$.

Lemma 2.5 ([39]). Let $\mathrm{U}$ be a partially ordered Banach space and $v_{n}, \mathrm{y}_{\mathrm{n}} \in \mathrm{V}$ such that $v_{\mathrm{n}} \leqslant \mathrm{y}_{\mathrm{n}}, \mathrm{n} \in \mathrm{Z}^{+}$. If $v_{n} \rightarrow v$ and $y_{n} \rightarrow y$, then $v \leqslant y$.

Assume that the following hypothesis hold:

$\left(C_{1}\right) k:[0,1] \times \mathbb{R} \rightarrow \mathbb{R}$ satisfies Carathéodory conditions;

$\left(C_{2}\right) k(t, v)$ is non decreasing in $v$ for each $t \in[0,1]$;

$\left(C_{3}\right)$ there exist constant $A>0$ such that $0 \leqslant k\left(t, v_{1}(t)\right)-k\left(t, v_{2}(t) \leqslant A\left(v_{1}-v_{2}\right)\right.$.

Lemma 2.6 ([1]). Let $v \in \mathrm{C}(0,1) \cap \mathrm{L}(0,1)$, then the following result holds

$$
\mathrm{I}^{\delta} \mathrm{D}^{\delta} v(\mathrm{t})=v(\mathrm{t})+\mathrm{c}_{1} \mathrm{t}^{\delta-1}+\mathrm{c}_{2} \mathrm{t}^{\delta-2}+\mathrm{c}_{3} \mathrm{t}^{\delta-3}+\cdots+\mathrm{c}_{\mathrm{m}} \mathrm{t}^{\delta-\mathrm{m}},
$$

where $c_{i} \in \mathbb{R}$, for $i=1,2, \ldots, m$, are constants.

\section{Main results}

This section is devoted to the main results.

Lemma 3.1. Under the assumption $\mathrm{h} \in \mathrm{C}([0,1], \mathbb{R})$, the following linear BVP of FDEs

$$
\mathrm{D}^{\mathrm{p}} v(\mathrm{t})+\mathrm{h}(\mathrm{t})=0, \mathrm{t} \in[0,1], 3<\mathrm{p} \leqslant 4, \quad v(0)=v^{\prime}(0)=v^{\prime \prime}(0)=v^{\prime \prime}(1)=0
$$

has a solution given by

$$
v(t)=\int_{0}^{1} \mathcal{H}(t, \rho) h(\rho) d \rho,
$$

where $\mathcal{H}(t, \rho)$ is the Green's function defined by

$$
\mathcal{H}(t, \rho)= \begin{cases}\frac{\mathrm{t}^{\mathrm{p}-1}(1-\rho)^{\mathrm{p}-3}-(\mathrm{t}-\rho)^{\mathrm{p}-1}}{\Gamma(p)}, & 0 \leqslant \rho \leqslant t \leqslant 1 \\ \frac{\mathrm{t}^{\mathrm{p}-1}(1-\rho)^{\mathrm{p}-3}}{\Gamma(p)}, & 0 \leqslant \mathrm{t} \leqslant \rho \leqslant 1 .\end{cases}
$$

Proof. We may apply Lemma 2.6 to reduce the equation (3.1) as

$$
v(t)=-I^{p} h(t)+c_{1} t^{p-1}+c_{2} t^{p-2}+c_{3} t^{p-3}+c_{4} t^{p-4} .
$$

Thank to boundary conditions $v(0)=v^{\prime}(0)=v^{\prime \prime}(0)=0$ we face to singularity. Therefore to avoid singularity, we take $c_{2}=c_{3}=c_{4}=0$. Inview of boundary condition $v^{\prime \prime}(1)=0$, we get $c_{1}=\frac{I^{p-2} h(1)}{(p-1)(p-2)}$. Hence, we deduce from (3.3) that

$$
v(t)=t^{p-1} \int_{0}^{1} \frac{(1-\rho)^{p-3}}{\Gamma(p)} h(\rho) d \rho-\int_{0}^{t} \frac{(t-\rho)^{p-1}}{\Gamma(p)} h(\rho) d \rho .
$$

Hence, we get from (3.4) as

$$
v(t)=\int_{0}^{1} \mathcal{H}(t, \rho) h(\rho) d \rho
$$

Clearly, $\mathcal{H}(t, \rho) \geqslant 0$ for all $t, \rho \in[0,1]$. 
Lemma 3.2. Thank to Lemma 3.1, the considered BVP (1.1) has a solution given by

$$
v(t)=\int_{0}^{1} \mathcal{H}(t, \rho) k(\rho, v(\rho)) d \rho,
$$

where $\mathcal{H}(t, \rho)$ is the Green's function defined in equation (3.2).

Lemma 3.3. The functions $\mathcal{H}(t, \rho)$ satisfies the following property

$$
\max _{\mathfrak{t} \in[0,1]} \int_{0}^{1} \mathcal{H}(\mathrm{t}, \rho) \mathrm{d} \rho \leqslant \frac{1}{(\mathrm{p}-2) \Gamma(\mathrm{p})}, \mathrm{t} \in[0,1] .
$$

Proof. Since $\mathcal{H}(\mathrm{t}, \rho)=\frac{\mathrm{t}^{\mathrm{p}-1}(1-\rho)^{\mathrm{p}-3}}{\Gamma(\mathrm{p})}-\frac{(\mathrm{t}-\rho)^{\mathrm{p}-1}}{\Gamma(\mathrm{p})} \leqslant \frac{\mathrm{t}^{\mathrm{p}-1}(1-\rho)^{\mathrm{p}-3}}{\Gamma(\mathrm{p})}$, it follows that

$$
\max _{t \in[0,1]} \int_{0}^{1} \mathcal{H}(t, \rho) d \rho \leqslant \frac{1}{(p-2) \Gamma(p)} .
$$

In the view of (3.5), we define an operator $\mathrm{T}: \mathrm{V} \rightarrow \mathrm{V}$ by

$$
\mathrm{T} v(\mathrm{t})=\int_{0}^{1} \mathcal{H}(\mathrm{t}, \rho) \mathrm{k}(\rho, v(\rho)) \mathrm{d} \rho .
$$

Then, in view of the integral equation (3.5), we may write integral equation (3.6) as

$$
(\mathrm{I}-\mathrm{T} v)(\mathrm{t})=0, \mathrm{t} \in[0,1] .
$$

It is obvious that the solution of equation (3.5) is the solution of equation (3.7), that is fixed point of the operator $\mathrm{T}$. Using $\left(\mathrm{C}_{2}\right)$, for $v, \mathrm{y} \in \mathrm{V}$ and $v \leqslant \mathrm{y}$, we obtain

$$
\mathrm{T} v(\mathrm{t})=\int_{0}^{1} \mathcal{H}(\mathrm{t}, \rho) k(\rho, v(\rho)) \mathrm{d} \rho \leqslant \int_{0}^{1} \mathcal{H}(t, \rho) k(\rho, y(\rho)) \mathrm{d} \rho=\mathrm{Ty}(\mathrm{t}),
$$

that is, $\mathrm{T}$ is nondecreasing.

Assume that

$\left(C_{4}\right) \alpha, \beta \in V$ are upper and lower solutions of (3.7) such that $\alpha \leqslant \beta$ on $[0,1]$.

Lemma 3.4. Under the assumptions $\left(\mathrm{C}_{1}\right)-\left(\mathrm{C}_{4}\right)$, there exists a monotone sequence of solution of the corresponding operator equation (3.7) which is converging to the solution of an integral equation (3.5) .

Proof. Let $\left(\mathrm{C}_{4}\right)$ holds, then, we can prove that the operator $\mathrm{T}$ is continuous and nondecreasing (in view of $\left(\mathrm{C}_{1}\right)$ and $\left.\left(\mathrm{C}_{2}\right)\right)$. Let $\nu_{1}, v_{2} \in \mathrm{V} \subset \mathrm{W}$,

$$
\begin{aligned}
\left|\mathrm{T} v_{1}(\mathrm{t})-\mathrm{T} v_{2}(\mathrm{t})\right| & =\left|\int_{0}^{1} \mathcal{H}(\mathrm{t}, \rho) \mathrm{k}\left(\rho, v_{1}(\rho)\right) \mathrm{d} \rho-\int_{0}^{1} \mathcal{H}(\mathrm{t}, \rho) \mathrm{k}\left(\rho, v_{2}(\rho)\right) \mathrm{d} \rho\right| \\
& \leqslant \int_{0}^{1} \mathcal{H}(\mathrm{t}, \rho) \mid \mathrm{k}\left(\rho, v_{1}(\rho)-\mathrm{k}\left(\rho, v_{2}(\rho)\right) \mid \mathrm{d} \rho \leqslant \frac{\mathrm{A}}{(\mathrm{p}-2) \Gamma(\mathrm{p})}\left\|v_{1}-v_{2}\right\| .\right.
\end{aligned}
$$

Thus in view of continuity of $k$ we have $T$ is a continuous function. It is easy to prove that the operator $T$ is uniformly bounded and equi-continuous. Under the Arzela Ascoli Theorem statement that "If $M$ be a family (finite or infinite) of an equi-continuous, uniformly bounded real valued functions $v$ on an interval $[0,1]$, then $M$ contains a uniformly convergent sequence of functions $v_{n}$, converging to a function $v$ in $W$ as $n \rightarrow \infty$, where $W$ denotes the space of all continuous bounded functions on $[0,1]$, thus any sequence 
in $V$ contains a uniformly bounded convergent subsequence on $[0,1]$ and consequently $V$ has a compact closure in $W^{\prime \prime}$. We apply the aforementioned theorem for operator $T$. Therefore $T$ is a compact operator by aforesaid theorem. We choose $v_{0}=\alpha$. In view of upper and lower solutions and the increasing property of operator $\mathrm{T}$, we have (in view of $\mathrm{C}_{4}$ ) that

$$
\begin{aligned}
\alpha & \leqslant \beta, \\
v_{0} & \leqslant \beta, \\
v_{0} \leqslant T v_{0} & \leqslant T \beta \leqslant \beta, \text { implies that } v_{0} \leqslant v_{1} \leqslant \beta \text { on }[0,1],
\end{aligned}
$$

where $v_{1}=\mathrm{T} v_{0}$ is an iterative solution of the operator equation (3.7). Again apply the operator $\mathrm{T}$ which gives

$$
\mathrm{T} v_{0} \leqslant \mathrm{~T} v_{1} \leqslant \mathrm{~T} \beta \leqslant \beta \text {, that implies, } v_{1} \leqslant v_{2} \leqslant \beta \text { on }[0,1],
$$

where $v_{2}=\mathrm{T} v_{1}$ is an iterative solution of the operator equation (3.7). According to aforementioned manner, we obtain

$$
v_{0} \leqslant v_{1} \leqslant v_{2} \leqslant \cdots \leqslant v_{n-1} \leqslant v_{n} \leqslant \beta \text { on }[0,1],
$$

which is a bounded monotone sequence $\left\{v_{n}\right\}$ of solutions of the operator equation (3.7). The boundedness and monotonicity of the sequence $\left\{v_{n}\right\}$ implies the existence of $v \in V$ so that $v_{n} \rightarrow v$ as $n \rightarrow \infty$. Hence, $v=\mathrm{T} v$, where $v$ is the solution of the integral equation (3.7) given by

$$
v(t)=\int_{0}^{1} \mathcal{H}(t, \rho) k(\rho, v(\rho)) d \rho, t \in[0,1] .
$$

Using the condition $\left(C_{3}\right)$, for any $v, y \in V$ and $v \leqslant y$ which implies that $k(t, v(t)) \leqslant k(t, y(t))$, we have

$$
\|\mathrm{T} y-\mathrm{T} v\| \leqslant \Delta\|y-v\|, \text { where } \Delta=\frac{A}{(p-2) \Gamma(p)} .
$$

Hence in view of (3.8) and (3.9), we get

$$
\begin{aligned}
\left\|v_{2}-v_{1}\right\| & =\left\|\mathrm{T} v_{1}-\mathrm{T} v_{0}\right\| \leqslant \Delta e_{1}, \\
\left\|v_{3}-v_{2}\right\| & =\left\|\mathrm{T} v_{2}-\mathrm{T} v_{1}\right\| \leqslant \Delta^{2} e_{1}, \\
\left\|v_{4}-v_{3}\right\| & =\left\|\mathrm{T} v_{3}-\mathrm{T} v_{2}\right\| \leqslant \Delta^{3} e_{1}, \\
& \vdots \\
\left\|v_{n+1}-v_{n}\right\| & =\left\|\mathrm{T} v_{n}-\mathrm{T} v_{n-1}\right\| \leqslant \Delta^{n} e_{1} .
\end{aligned}
$$

Thus for every positive integer $m, n$, one can obtain

$$
\left\|v_{m+n}-v_{n}\right\| \leqslant\left\|v_{n+m}-v_{n+m-1}\right\|+\left\|v_{n+m-1}-v_{n+m-2}\right\|+\cdots+\left\|v_{n+1}-v_{n}\right\| \leqslant \Delta^{n} \frac{1-\Delta^{m}}{1-\Delta} e_{1} .
$$

As $\Delta<1, \mathrm{n} \rightarrow \infty$ implies that $\left\|v_{\mathrm{m}+\mathrm{n}}-v_{\mathrm{n}}\right\| \rightarrow 0$. Thus $\left\{v_{\mathrm{n}}\right\}$ in $\mathrm{V}$ is a Cauchy sequence. Let $v^{*}(\mathrm{t})=$ $\lim _{n \rightarrow \infty} v_{n}(t)$, thus $T v^{*}=v^{*}$. Apply $m \rightarrow \infty$ in (3.10), then, we obtain the following estimate of error for lower solution,

$$
e_{n}=\left\|v^{*}-v_{n}\right\| \leqslant \frac{\Delta^{n}}{1-\Delta} e_{1}, \text { where } e_{1}=\left\|v_{1}-v_{0}\right\| .
$$

Remark 3.5. If we choose $v_{0}=\beta$, then we obtain a monotone bounded decreasing sequence $\left\{v_{n}\right\}$ so that

$$
v_{0} \geqslant v_{1} \geqslant v_{2} \geqslant \cdots \geqslant v_{n-1} \geqslant v_{n} \geqslant \alpha \text { on }[0,1],
$$

which is converging to the solution of the problem (3.5). Similarly, for maximum error estimate for maximal solutions of the problem (1.1), one can easily obtain the following: $e_{n}^{*}=\left\|v_{n}^{*}-\bar{v}^{*}\right\| \leqslant \frac{\Delta^{n}}{1-\Delta} e_{1}^{*}$, where $e_{1}^{*}=$ 
$\left\|v_{0}^{*}-v_{1}^{*}\right\|$. According to the afore proved Lemma 3.4, the iterative sequences for the nonlinear fractional differential equation (1.1) are constructed as

$$
v_{n}(t)=\int_{0}^{1} \mathcal{H}(t, \rho) k\left(\rho, v_{n-1}\right) d \rho, n \geqslant 1, \quad v_{n}^{*}(t)=\int_{0}^{1} \mathcal{H}(t, \rho) k\left(\rho, v_{n-1}^{*}\right) d \rho, n \geqslant 1,
$$

from which, we have $v^{*}(\mathrm{t})=\lim _{n \rightarrow \infty} v_{\mathfrak{n}}(\mathrm{t}), \bar{v}^{*}(\mathrm{t})=\lim _{\mathrm{n} \rightarrow \infty} v_{\mathrm{n}}^{*}(\mathrm{t})$.

Theorem 3.6. Let consider the assumptions $\left(\mathrm{C}_{1}\right),\left(\mathrm{C}_{2}\right)$, and $\left(\mathrm{C}_{3}\right)$, and $\Delta<1$, then the BVP (1.1) has unique minimal and maximal solutions.

Proof. The proof is obvious and straight forward by using the hypothesis $\left(C_{1}\right),\left(C_{2}\right)$, and $\left(C_{3}\right)$, so we omit it.

\section{Ulam type stability analysis of BVP (1.1)}

In this section, we develop sufficient conditions for the Ulam stability analysis of the solutions to the considered BVP of FDEs (1.1). The required definitions and lemmas can be found in [3, 5, 9, 21, 26$28,34,35]$.

Definition 4.1. The BVP (1.1) is said to be Ulam-Hyers stable if there exists $\mathbf{c}_{k} \in \mathbb{R}^{+}$such that for every $\varepsilon>0$ and for every solution $v \in C^{4}([0,1], \mathbb{R})$ of the inequality

$$
\left|D^{p} v(t)+k(t, v(t))\right| \leqslant \varepsilon, t \in[0,1],
$$

there exists a unique solution $\bar{v} \in \mathrm{C}^{4}([0,1], \mathbb{R})$ of the equation (1.1), such that

$$
|v(t)-\bar{v}(t)| \leqslant \mathbf{c}_{k} \varepsilon, t \in[0,1] .
$$

Definition 4.2. The BVP of FDEs (1.1) is said to be generalized Ulam-Hyers stable if there exists $\varphi \in$ $\mathrm{C}\left(\mathbb{R}^{+}, \mathbb{R}^{+}\right), \varphi(0)=0$, such that for every solution $v \in \mathrm{C}^{4}\left([0,1], \mathbb{R}^{+}\right)$of the inequality (4.1), there exists a unique solution $\bar{v} \in \mathrm{C}^{4}\left([0,1], \mathbb{R}^{+}\right)$of the considered problem (1.1), such that

$$
|v(t)-\bar{v}(t)| \leqslant \phi(t) \varepsilon, t \in[0,1] .
$$

Definition 4.3. The BVP (1.1) is said to be Ulam-Hyers-Rassias stable with respect to $\phi \in C\left([0,1], \mathbb{R}^{+}\right)$, if there exists a non zero positive real number $\mathbf{c}_{k}$, such that for every $\varepsilon>0$ and for every solution $v \in \mathrm{C}^{4}([0,1], \mathbb{R})$ of the inequality

$$
\left|D^{p} v(t)+k(t, v(t))\right| \leqslant \varepsilon \phi(t), t \in[0,1],
$$

there exists a unique solution $\bar{v} \in C^{4}([0,1], \mathbb{R})$ of the considered problem (1.1), such that

$$
|v(t)-\bar{v}(t)| \leqslant \mathbf{c}_{k} \varepsilon \phi(t), t \in[0,1] .
$$

Definition 4.4. The BVP (1.1) is said to be generalized Ulam-Hyers-Rassias stable with respect to $\phi \in$ $\mathrm{C}([0,1], \mathbb{R})$, if there exists $\mathbf{c}_{\phi} \in \mathbb{R}^{+}$, such that for every solution $v \in \mathrm{C}^{1}([0,1], \mathbb{R})$ of the inequality

$$
\left|D^{p} v(t)+k(t, v(t))\right| \leqslant \phi(t), t \in[0,1],
$$

there exists a unique solution $\bar{v} \in C^{4}([0,1], \mathbb{R})$ of the considered problem (1.1), such that

$$
|v(t)-\bar{v}(t)| \leqslant \mathbf{c}_{k} \phi(t), t \in[0,1] .
$$

Remark 4.5. A function $v \in C^{4}([0,1], \mathbb{R})$ is a solution of the inequality (4.1) if there exists a function $\psi \in \mathrm{C}([0,1], \mathbb{R})$ (dependent on $v)$, such that 
(I) $\mathrm{D}^{\mathrm{p}} v(\mathrm{t})+\mathrm{k}(\mathrm{t}, v(\mathrm{t}))=\psi(\mathrm{t}), \mathrm{t} \in[0,1]$;

(II) $|\psi(t)| \leqslant \varepsilon$, for all $t \in[0,1]$.

Theorem 4.6. Under the hypothesis $\left(C_{2}\right)$ and $\left(C_{3}\right)$ coupled with the condition $(p-2) \Gamma(p)>A$, the solutions of the BVP (1.1) are Ulam-Hyers stable and consequently generalized Ulam-Hyers stable.

Proof. Assume that $\left(C_{2}\right)$ and $\left(C_{3}\right)$ coupled with the condition $\Gamma(p+1)>A$ hold. Let $v \in C^{4}([0,1], \mathbb{R})$ be any solution of the inequality $(4.1)$ and $\bar{v} \in \mathrm{C}^{4}([0,1], \mathbb{R})$ be a unique solution of the BVP

$$
\mathrm{D}^{\mathrm{p}} v(\mathrm{t})+\mathrm{k}(\mathrm{t}, v(\mathrm{t}))=\psi(\mathrm{t}) \text { for all } \mathrm{t} \in[0,1], 3<\mathrm{p} \leqslant 4 .
$$

Thank to Lemma 3.1, we obtain

$$
v(t)=\int_{0}^{1} \mathcal{H}(t, \rho) k(\rho, v(\rho)) \mathrm{d} \rho+\int_{0}^{1} \mathcal{H}(t, \rho) \psi(\rho) \mathrm{d} \rho, \text { where } \psi \in \mathrm{C}([0,1], \mathbb{R}),
$$

which yields

$$
\left|v(t)-\int_{0}^{1} \mathcal{H}(t, \rho) k(\rho, v(\rho)) d \rho\right| \leqslant \frac{\varepsilon}{(p-2) \Gamma(p)}, t \in[0,1] .
$$

Using the inequality (4.2) and Remark 4.5, we get the stability result for solutions for $t \in[0,1]$ as

$$
\begin{aligned}
|v(t)-\bar{v}(t)| & =\left|v(t)-\int_{0}^{1} \mathcal{H}(t, \rho) \mathrm{k}(\rho, \bar{v}(\rho)) \mathrm{d} \rho\right| \\
& =\left|v(\mathrm{t})-\int_{0}^{1} \mathcal{H}(\mathrm{t}, \rho) \mathrm{k}(\rho, v(\rho)) \mathrm{d} \rho+\int_{0}^{1} \mathcal{H}(\mathrm{t}, \rho) \mathrm{k}(\rho, v(\rho)) \mathrm{d} \rho-\int_{0}^{1} \mathcal{H}(\mathrm{t}, \rho) \mathrm{k}(\rho, \bar{v}(\rho)) \mathrm{d} \rho\right| \\
& \leqslant\left|v(\mathrm{t})-\int_{0}^{1} \mathcal{H}(\mathrm{t}, \rho) \mathrm{k}(\rho, v(\rho)) \mathrm{d} \rho\right|+\int_{0}^{1}|\mathcal{H}(\mathrm{t}, \rho)| \mathrm{k}(\rho, v(\rho))-\mathrm{k}(\rho, \bar{v}(\rho)) \mid \mathrm{d} \rho,
\end{aligned}
$$

which implies that

$$
\|v-\bar{v}\| \leqslant \frac{\varepsilon}{(p-2) \Gamma(p)}+\frac{A}{(p-2) \Gamma(p)}\|v-\bar{v}\|
$$

on simplification it gives

$$
\|v-\bar{v}\| \leqslant \frac{\varepsilon}{(p-2) \Gamma(p)-A}, \text { where } c_{k}=\frac{1}{(p-2) \Gamma(p)-A} .
$$

Hence the solutions of BVP (1.1) are Ulam-Hyers stable. By putting $\phi(\varepsilon)=\mathbf{c}_{k} \varepsilon, \phi(0)=0$, In view of this we have the conclusion that the solutions of BVP (1.1) are generalized Ulam-Hyers stable.

Remark 4.7. A function $v \in C^{3}([0,1], \mathbb{R})$ is a solution of the inequality (4.4) if there exists a function $\psi \in \mathrm{C}([0,1], \mathbb{R})$ (dependent on $v$ ), such that

(I) $\mathrm{D}^{\mathrm{p}} v(\mathrm{t})+\mathrm{k}(\mathrm{t}, v(\mathrm{t}))=\psi(\mathrm{t}), \mathrm{t} \in[0,1]$;

(II) $|\psi(t)| \leqslant \varepsilon \phi(t)$ for all $t \in[0,1]$, where $\phi \in C([0,1], \mathbb{R})$.

Due to Remark 4.7, we get the solution of

$$
D^{p} v(t)+k(t, v(t))=\psi(t), t \in[0,1], \quad v(0)=v^{\prime}(0)=v^{\prime \prime}(0)=v^{\prime \prime}(1)=0
$$

by using Lemma 3.1 as

$$
v(t)=\int_{0}^{1} \mathcal{H}(t, \rho) k(\rho, v(\rho)) \mathrm{d} \rho+\int_{0}^{1} \mathcal{H}(t, \rho) \psi(\rho) \mathrm{d} \rho, \text { where } \psi \in \mathrm{C}([0,1], \mathbb{R}),
$$

which yields

$$
\left|v(t)-\int_{0}^{1} \mathcal{H}(t, \rho) k(\rho, v(\rho)) d \rho\right| \leqslant \frac{\varepsilon}{(p-2) \Gamma(p)} \phi(t), t \in[0,1]
$$


Theorem 4.8. Assume that $\left(\mathrm{C}_{2}\right)$ and $\left(\mathrm{C}_{3}\right)$ along with the condition $(\mathrm{p}-2) \Gamma(\mathrm{p})>A$ hold, then the solutions of BVP (1.1) are Ulam-Hyers-Rassias stable. Further the solutions of the considered BVP (1.1) are generalized Ulam-Hyers-Rassias stable.

Proof. Let for every $\mathrm{t} \in[0,1]$, if $v \in \mathrm{C}^{4}([0,1], \mathbb{R})$ be any solution of the inequality $(4.3)$ and $\bar{v} \in \mathrm{C}^{4}([0,1], \mathbb{R})$ is the unique solution of BVP (1.1), then using Remark 4.7, we have

$$
\begin{aligned}
|v(t)-\bar{v}(t)| & =\left|v(t)-\int_{0}^{1} \mathcal{H}(t, \rho) k(\rho, \bar{v}(\rho)) \mathrm{d} \rho\right| \\
& =\left|v(t)-\int_{0}^{1} \mathcal{H}(t, \rho) k(\rho, v(\rho)) \mathrm{d} \rho+\int_{0}^{1} \mathcal{H}(t, \rho) k(\rho, v(\rho)) \mathrm{d} \rho-\int_{0}^{1} \mathcal{H}(t, \rho) k(\rho, \bar{v}(\rho)) \mathrm{d} \rho\right| \\
& \leqslant\left|v(t)-\int_{0}^{1} \mathcal{H}(t, \rho) k(\rho, v(\rho)) \mathrm{d} \rho\right|+\int_{0}^{1}|\mathcal{H}(t, \rho)| k(\rho, v(\rho))-k(\rho, \bar{v}(\rho)) \mid \mathrm{d} \rho .
\end{aligned}
$$

Which implies that

$$
\|v-\bar{v}\| \leqslant \frac{\varepsilon \phi(t)}{(p-2) \Gamma(p)}+\frac{A}{(p-2) \Gamma(p)}\|v-\bar{v}\|,
$$

on simplification it gives

$$
\|v-\bar{v}\| \leqslant \varepsilon \mathbf{c}_{k} \phi(t), \text { where } \mathbf{c}_{k}=\frac{1}{(p-2) \Gamma(p)-A} .
$$

Therefore the solutions of BVP (1.1) are Ulam-Hyers-Rassias stable. By putting $\varphi(\varepsilon)=\varepsilon \phi(t), \varphi(0)=0$. Analogously one can easily prove hat the solutions of BVP (1.1) are generalized Ulam-Hyers-Rassias stable.

\section{Examples}

\section{Example 5.1.}

$$
\left\{\begin{array}{l}
\mathrm{D}^{\frac{7}{2}} v(\mathrm{t})+\mathrm{t}+v^{0.5}(\mathrm{t})=0, \mathrm{t} \in[0,1], \\
v(0)=v^{\prime}(0)=v^{\prime \prime}(0)=v^{\prime \prime}(1)=0,
\end{array}\right.
$$

where $k(t, v(t))=t+v^{0.5}(t)$. On computation $A=0.00001$, from which it is easy to see that $\Delta=$ $\frac{A}{(p-2) \Gamma(p)}=2.0060 e^{-06}<1$. When $n=3$ is large enough. Therefore, we take the iterative sequences for approximate minimal and maximal solutions $\bar{v}_{\mathrm{n}}$ and $\bar{v}_{\mathrm{n}}^{*}$ as

$$
v^{*}(t)=v_{3}(t)=\int_{0}^{1} \mathcal{H}(t, \rho) k\left(\rho, v_{2}(\rho)\right) d \rho, \quad \bar{v}^{*}(t)=v_{3}^{*}(t)=\int_{0}^{1} \mathcal{H}(t, \rho) k\left(\rho, v_{2}^{*}(\rho)\right) d \rho,
$$

respectively. Let $v_{0}=-1, v_{0}^{*}=10$ be maximal and minimal solutions of the BVP (5.1) respectively, then

$$
\begin{aligned}
& e_{3}=\left\|v(t)-v_{3}(t)\right\| \leqslant \frac{\Delta^{3}}{1-\Delta} \times e_{1} \leqslant 8.07232184169 e^{-18} \times \max _{t \in[0,1]}\left|v_{1}(t)+1\right| \simeq 9.04509912854 e^{-18}, \\
& e_{3}^{*}=\left\|v(t)-v_{3}^{*}(t)\right\| \leqslant \frac{\Delta^{3}}{1-\Delta} \times e_{1}^{*} \leqslant 8.07232184169 e^{-18} \times \max _{t \in[0,1]}\left|10-v_{1}^{*}(t)\right| \simeq 8.07232184169 e^{-17} .
\end{aligned}
$$

In the given Figure 1, we provide the plot of approximate, minimal, and maximal solutions.

Clearly $A<(p-2) \Gamma(p)$, from which different kinds of stability results can be easily verified. Hence the solutions of BVP (5.1) are Ulam-Hyers, generalized Ulam Hyers, Ulam-Hyers-Rassias and generalized Ulam-Hyers-Rassias stable. 

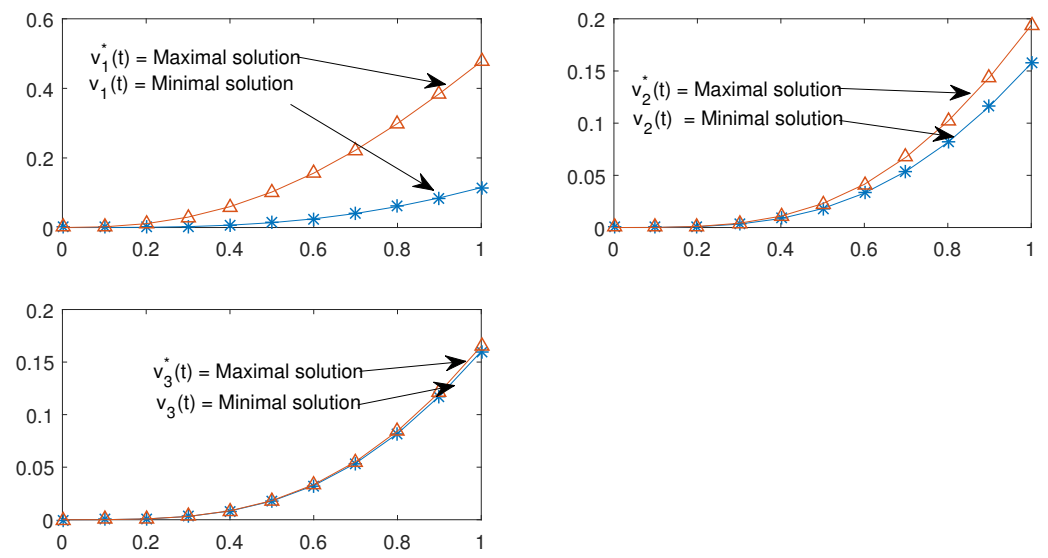

Figure 1: A plot of maximal and minimal solutions of Example 5.1.

\section{Example 5.2.}

$$
\left\{\begin{array}{l}
D^{\frac{10}{3}} v(t)+v^{\frac{2}{3}}(t)(1-t) e^{-v(t)}=0, t \in[0,1], \\
v(0)=v^{\prime}(0)=v^{\prime \prime}(0)=v^{\prime \prime}(1)=0,
\end{array}\right.
$$

where $k(t, v(t))=v^{\frac{2}{3}}(t)(1-t) e^{-v(t)}$. Let $A=0.00001$, it is easy to see that $\Delta=\frac{A}{(p-2) \Gamma(p)}=2.6996 e^{-06}<$ 1. When $n=3$ is large enough . Therefore, we can obtain the iterative sequences for approximate minimal and maximal solutions $\bar{v}_{\mathrm{n}}$ and $\bar{v}_{\mathrm{n}}^{*}$ as given by

$$
v^{*}(t)=v_{3}(t)=\int_{0}^{1} \mathcal{H}(t, \rho) k\left(\rho, v_{2}(\rho)\right) d \rho, \quad \bar{v}^{*}(t)=v_{3}^{*}(t)=\int_{0}^{1} \mathcal{H}(t, \rho) k\left(\rho, v_{2}^{*}(\rho)\right) d \rho,
$$

respectively. Let $v_{0}=-0.1, v_{0}^{*}=0.1$ be maximal and minimal solutions respectively of the BVP (5.2), then

$$
\begin{aligned}
& e_{3}=\left\|v(t)-v_{3}(t)\right\| \leqslant \frac{\Delta^{3}}{1-\Delta} \times e_{1} \leqslant 1.96749624928 e^{-17} \times \max _{t \in[0,1]}\left|v_{1}(t)+0.1\right| \simeq 1.96749624928 e^{-18,}, \\
& e_{3}^{*}=\left\|v(t)-v_{3}^{*}(t)\right\| \leqslant \frac{\Delta^{3}}{1-\Delta} \times e_{1}^{*} \leqslant 1.96749624928 e^{-17} \times \max _{t \in[0,1]}\left|0.1-v_{1}^{*}(t)\right| \simeq 1.96749624928 e^{-18} .
\end{aligned}
$$

The plot of approximate, maximal, and minimal solutions is given in Figure 2.
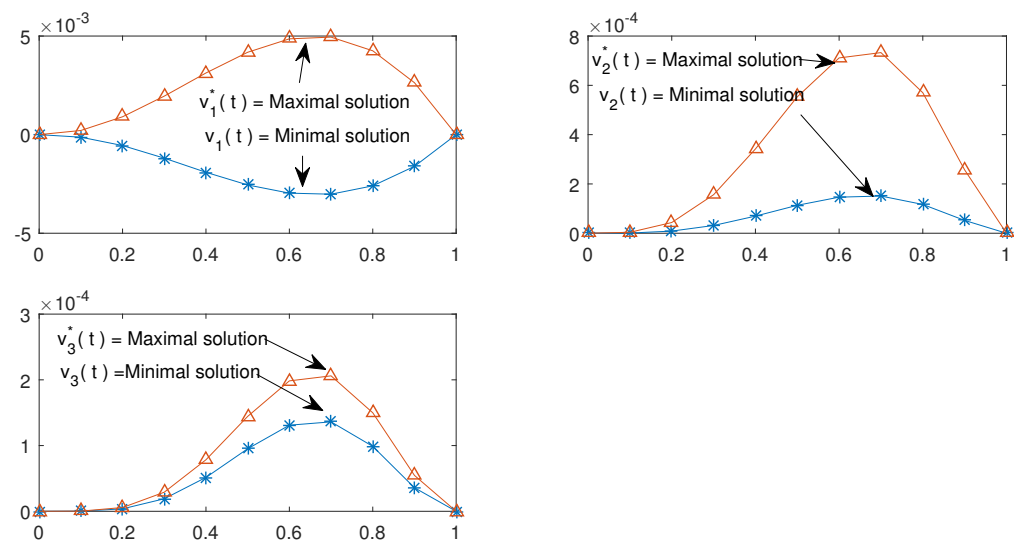

Figure 2: A plot of maximal and minimal solutions of Example 5.2. 
It is obvious that $A<(p-2) \Gamma(p)$, from which different kinds of stability results can be easily verified. Hence the solutions of BVP (5.2) are Ulam-Hyers, generalized Ulam Hyers, Ulam-Hyers-Rassias, and generalized Ulam-Hyers-Rassias stable. The stable behaviors can be observed from Figure 2.

\section{Conclusion}

We have successfully established sufficient conditions for the existence of minimal and maximal solutions to boundary value problems of fractional order differential equations. The iterative approximate solutions are obtained of the boundary value problems of NFDEs via monotone iterative method. The obtained iterative approximate solutions of minimal and maximal solutions to the BVP (1.1) are also plotted via MATLAB software. The clear presentation of the iterative solutions in the plots illustrates that the monotone iterative technique is applicable and very efficient for the BVP of NFDEs. Furthermore, various type of stability have been investigated for the proposed BVP (1.1). We have also provided the maximum error estimates of each illustrative example of the proposed boundary value problems of NFDEs.

\section{Acknowledgment}

We all authors have read and approved the final version.

\section{References}

[1] R. P. Agarwal, M. Belmekki, M. Benchohra, A survey on semilinear differential equations and inclusions involving Riemann-Liouville fractional derivative, Adv. Difference Equ., 2009 (2009), 47 pages. 1, 2.6

[2] R. P. Agarwal, M. Benchohra, S. Hamani, A survey on existence results for boundary value problems of nonlinear fractional differential equations and inclusions, Acta Appl. Math., 109 (2012), 973-1033. 1

[3] N. Ahmad, Z. Ali, K. Shah, A. Zada, Analysis of implicit type nonlinear dynamical problem of impulsive fractional differential equation, Complexity, 2018 (2018), 15 pages. 4

[4] B. Ahmad, J. J. Nieto, Existence results for a coupled system of nonlinear fractional differential equations with three-point boundary conditions, Comput. Math. Appl., 58 (2009), 1838-1843. 1

[5] N. Ali, B. B. Fatima, K. Shah, R. A. Khan, Hyers-Ulam Stability of a Class of Nonlocal Boundary Value Problem Having Triple Solutions, Hyers-Ulam stability of a class of nonlocal boundary value problem having triple solutions, Int. J. Appl. Comput. Math., 4 (2018), 12 pages. 4

[6] M. Al-Refai, M. Ali Hajji, Monotone iterative sequences for nonlinear boundary value problems of fractional order, Nonlinear Anal., 74 (2011), 3531-3539. 1

[7] C.-Z. Bai, J.-X. Fang, The existence of a positive solution for a singular coupled system of nonlinear fractional differential equations, Appl. Math. Comput., 150 (2004), 611-621. 1

[8] M. Benchohra, J. R. Graef, S. Hamani, Existence results for boundary value problems with nonlinear fractional differential equations, Applicable Anal., 87 (2008), 851-863. 1

[9] S. Bushnaq, W. Hussain, K. Shah, On nonlinear implicit fractional differential equations without compactness, J. Nonlinear Sci. Appl., 10 (2017), 5528-5539. 4

[10] A. Cabadal, G. T. Wang, Positive solutions of nonlinear fractional differential equations with integral boundary value conditions, J. Math. Anal. Appl., 389 (2012), 403-411.

[11] C. De Coster, P. Habets, Two-point Boundary Value Problems: Lower and Upper Solutions, Elsevier B. V., Amsterdam, (2006).

[12] V. Gafiychuk, B. Datsko, V. Meleshko, D. Blackmore, Analysis of the solutions of coupled nonlinear fractional reactiondiffusion equations, Chaos Solitons Fractals, 41 (2009), 1095-1104. 1

[13] Z. M. He, X. M. He, Monotone iterative technique for impulsive integro-differential equations with periodic boundary conditions, Comput. Math. Appl., 48 (2004), 73-84.

[14] R. Hilfer, Applications of Fractional Calculus in Physics, World Scientific Publishing Co., River Edge, (2000). 1, 2

[15] H. Khalil, K. Shah, R. A. Khan, Upper and lower solutions to a coupled system of nonlinear fractional differential equations, Progress in Fractional Differential Equations Applications, 2016 (2016), 10 pages. 1

[16] R. A. Khan, Existence and approximation of solutions to three-point boundary value problems for fractional differential equations, Electron. J. Qual. Theory Differ. Equ., 2011 (2011), 8 pages. 1

[17] A. A. Kilbas, H. M. Srivastava, J. J. Trujillo, Theory and Applications of Fractional Differential Equations, Elsevier Science B.V., Amsterdam, (2006). 1, 2

[18] G. S. Ladde, V. Lakshmikantham, A. S. Vatsala, Monotone iterative technique for nonlinear differential equations, Pitman Publishing Inc., Boston, (1985). 1 
[19] F. Li, M. Jia, X. Liu, C. Li, G. Li, Existence and uniqueness of solutions of secend-order three-point boundary value problems with upper and lower solutions in the reverse order, Nonlinear Anal., 68 (2008), 2381-2388.

[20] F. F. Li, J. T. Sun, M. Jia, Monotone iterative method for the second-order three-point boundary value problem with upper and lower solutions in the reversed order, Comput. Math. Appl., 217 (2011), 4840-4847.

[21] M. Li, J. R. Wang, D. O'Regan, Existence and Ulam's stability for conformable fractional differential equations with constant coefficients, Bull. Malays. Math. Sci. Soc., 2017 (2017), 1-22. 1, 4

[22] X. P. Liu, M. Jia, Multiple solutions for fractional differential equations with nonlinear boundary conditions, Comput. Math. Appl., 59 (2010), 2880-2886.

[23] F. A. McRae, Monotone iterative technique and existence results for fractional differential equations, Nonlinear Anal., 71 (2009), 6093-6096. 1

[24] K. S. Miller, B. Ross, An Introduction to the Fractional Calculus and Fractional Differential Equations, John Wiley \& Sons, New York, (1993). 1, 2

[25] I. Podlubny, Fractional Differential Equations: An introduction to fractional derivatives, fractional differential equations, to methods of their solution and some of their applications, Academic Press, San Diego, (1999). 1, 2

[26] T. M. Rassias, On the stability of the linear mapping in Banach spaces, Proc. Amer. Math. Soc., 72 (1978), 297-300. 1, 4

[27] T. M. Rassias, On the stability of functional equations and a problem of Ulam, Acta Appl. Math., 62 (2000), $23-130$.

[28] I. A. Rus, Ulam stabilities of ordinary differential equations in a Banach space, Carpathian J. Math., 26 (2010), $103-107$. 1,4

[29] S. G. Samko, A. A. Kilbas, O. I. Marichev, Fractional Integrals and Derivatives: Theory and Applications, Gordon and Breach Science Publishers, Yverdon, (1993). 1

[30] K. Shah, R. A. Khan, Iterative solutions to a coupled system of nonlinear fractional differential equations, J. Fract. Cal. Appl., 7 (2016), 40-50. 1

[31] K. Shah, R. A. Khan, Iterative scheme for a coupled system of fractional-order differential equations with three-point boundary conditions, Math. Methods Appl. Sci., 41 (2018), 1047-1053. 1

[32] X. Su, Boundary value problem for a coupled systemof nonlinear fractional differential equations, Appl. Math. Lett., 22 (2009), 64-69. 1

[33] G. T. Wang, R. P. Agarwal, A. Cabada, Existence results and the monotone iterative technique for systems of nonlinear fractional differential equations, Appl. Math. Lett., 25 (2012), 1019-1024. 1, 2

[34] J. R. Wang, M. Feckan, Y. Zhou, Fractional order differential switched systems with coupled nonlocal initial and impulsive conditions, Bull. Sci. math., 141 (2017), 727-746. 1, 4

[35] J. R. Wang, K. Shah, A. Ali, Existence and Hyers-Ulam stability of fractional nonlinear impulsive switched coupled evolution equations, Math. Methods Appl. Sci., 41 (2018), 2392-2402. 4

[36] J. H. Wang, H. J. Xiang, Z. G. Liu, Positive solution to nonzero boundary values problem for a coupled system of nonlinear fractional differential equations, Int. J. Differ. Equ., 2010 (2010), 12 pages. 1

[37] N. Xu, W. B. Liu, Iterative solutions for a coupled system of fractional differential-integral equations with two-point boundary conditions, Appl. Math. Comput., 244 (2014), 903-911. 1, 2

[38] W. G. Yang, Positive solutions for a coupled system of nonlinear fractional differential equations with integral boundary conditions, Comput. Math. Appl., 63 (2012), 288-297. 1

[39] E. Zeidler, Nonlinear Functional Analysis and its Applications: II/B: Nonlinear Monotone Operators, Springer-Verlag, New York, (1990). 1, 2, 2.5

[40] S. Q. Zhang, Monotone iterative method for initial value problem involving Riemann-Liouville fractional derivatives, Nonlinear Anal., 71 (2009), 2087-2093. 1 